\title{
Insulin pump therapy in type 1 diabetes mellitus
}

\author{
Raphael Del Roio Liberatore Jr., ${ }^{1}$ Durval Damiani²
}

\begin{abstract}
Objective: To review the current experience with insulin pump therapy in children and adolescents in order to guide pediatricians regarding indications and complications.

Sources of data: Systematic review of articles published in the literature referring to the use of insulin pump therapy, indications, complications and response to treatment. All articles published between 1995 and 2005 and appearing in the MEDLINE and LILACS databases were reviewed. The keywords were: insulin pump, type 1 diabetes mellitus and diabetes mellitus. The articles covering the subject of interest and referring to children and adolescents were selected.

Summary of the findings: Insulin pump therapy is not required for all patients with type 1 diabetes, since intensive treatments produce very similar results in terms of glycated hemoglobin and control of complications over the medium and long terms. However, the pump allows for greater comfort for patients, with less rigid meal schedules and better quality of life. The first requirement for patients intending to use the pump is getting used to having a device attached to the body and following strict glucose control; otherwise, pump therapy is not advantageous. Complications are rare due to the technologies currently available. The cost, however, is greater than with conventional treatments.

Conclusion: The development of infusion pumps and glucose monitors, including continuous monitoring systems, will lead to "intelligent pumps," so that a true "artificial pancreas" will be available, which can even be implanted in the patient, allowing non-diabetic persons to lead a normal life.
\end{abstract}

J Pediatr (Rio J). 2006;82(4):249-54: Type 1 diabetes mellitus, insulin therapy, insulin pump infusion, intensive treatment, glucose monitoring.

\section{Introduction}

With the progressive increase in insulin pump therapy usage by diabetic patients, pediatricians are often faced with patients treated with an "instrument" that is foreign to them. The present article aims to present the basic concepts of insulin pump therapy, which may be wellknown to endocrinologist/diabetologists but are probably not familiar to general pediatricians, and even to endocrinologists who do not treat diabetes.

During the 1990s, two studies showed that good metabolic control promotes a dramatic reduction in the

1. Professor coordenador, Doutor. Chefe, Serviço de Endocrinologia Pediátrica, Departamento de Pediatria e Cirurgia Pediátrica, Faculdade de Medicina de São José do Rio Preto, São José do Rio Preto, SP, Brasil.

2. Professor livre-docente. Chefe, Unidade de Endocrinologia Pediátrica, Instituto da Criança, Hospital das Clínicas, Faculdade de Medicina, Universidade de São Paulo (USP), São Paulo, SP, Brasil.

Manuscript received Mar 06 2006, accepted for publication Apr 262006.

Suggested citation: Liberatore Jr. RR, Damiani D. Insulin pump therapy in type 1 diabetes mellitus. J Pediatr (Rio J). 2006;82:249-54. incidence and development of diabetes-related complications. The Diabetes Control and Complications Trials (DCCT), including type 1 diabetes patients, showed that reductions in the levels of glycated hemoglobin (A1c) were associated with important reductions in the incidence and evolution of diabetic ${ }^{1}$ nephropathy and retinopathy. The United Kingdom Prospective Diabetes Study (UKPDS) involving patients with type 2 diabetes, reached similar, although less striking, results. 1,2

Some of the intensive therapy diabetic patients in the DCCT used insulin pump therapy, with slightly improved results for glycated hemoglobin, however with larger incidence of metabolic ketoacidosis. The age range in this group of patients was 13 to 20 years. ${ }^{1}$

Therapy with insulin pump infusion has been growing considerably in the last years, mainly in North America and Europe, and it is estimated that about $8 \%$ of the diabetic population over 20 years of age, in the USA, is using that therapeutic modality for treatment of diabetes. That percentage represents about 70,000 people in the adult 
age group alone. ${ }^{3}$ The numbers are not known for the pediatric age group, but the popularity of this therapy to treat diabetes has also been growing among children and adolescents. ${ }^{3,4}$

The aim of this article is to review the experience in the use of insulin pump therapy in children and adolescents.

\section{History and pump functioning}

The first insulin pumps appeared in the end of the 1970 s and were experimentally used in children at Yale University. The early pumps were large and heavy, and their purpose was to simulate the function of the pancreas by continuous subcutaneous insulin infusion. 5,6

In the mid 1980 s, a national experience, resulting from an adapted desferrioxamine infusion pump (Desferal ${ }^{\circledR}$ ), was tested.

In the course of time and especially in the 1990s, with improving technology, infusion pumps became smaller and more reliable, providing the combination of several infusion 7 speeds.

Currently, there are three manufactures of infusion pumps, Disetronic Medical Systems, MiniMed Technologies and Animas. The different brands are similar in terms of operation, size and weight, with variations referring mainly to appearance and use of control buttons. The three brands infuse insulin from a reservoir through a catheter inserted into the subcutaneous tissue via a small needle. The type of catheter, subcutaneous implantation and other small details may vary.

The pumps weigh about $400 \mathrm{~g}$ and their size is approximately that of a pager. The catheter can be disconnected from the pump for bathing or even for other water activities, such as swimming. Other sports can be practiced with the pump connected or having the continuous operation interrupted. 8

The infusion system should be changed periodically ( 3 to 5 days) to avoid allergic reactions and/or infection at the place of catheter insertion. Even in the absence of infection, the permanence of the infusion system for longer periods reduces therapeutic effectiveness, due to the gradual obstruction of infusion and worsening of metabolic control.

The catheter is always implanted in the subcutaneous tissue, but with a rotation of implantation sites. Generally, any place with sufficient subcutaneous tissue can be used. However, the most frequent sites are: abdominal wall, around the umbilicus, upper buttocks, and occasionally the thighs. A decisive factor for choosing the insertion site infusion catheter length. The infusion catheters are made of flexible plastic material, with two different lengths, 60 and $110 \mathrm{~cm}$. The catheters are connected to an infusion cannula that will be implanted under the skin, with the use of a metallic guide. There are two implantation forms: $90^{\circ}$ or angled. The insertion needle varies in length from 6 or 7 , to $17 \mathrm{~mm}$ depending on the type of insertion.

The cannula size influences the depth of the implant. Ultimately, the choice will depend on the amount of subcutaneous tissue available at the infusion site. The catheter size depends on the user size and, therefore, on the distance between the infusion site and the pump.

Regular insulin and ultrafast insulin (lispro/aspart) can be used for pump infusion, with no need for dilution. Although the initial studies report positive results with regular insulin, more recent results show advantages in the use of fast action insulin due to its quicker action, early peak, more predictable absorption and lower risk of catheter obstruction by insulin ${ }^{9-13}$ crystals.

\section{Introducing the patient to the pump}

Initially, it was believed that only well-trained individuals approaching the end of adolescence, and who were, above all, motivated, should receive insulin ${ }^{14}$ pump therapy. Nowadays, this therapeutic modality has proved particularly useful in patients with difficult-tocontrol diabetes, who present repeated episodes of metabolic acidosis or hypoglycemia. Therefore, differently than was initially thought, this treatment modality may be more useful with "problem-patients" or difficult-to-control diabetes. ${ }^{15-17}$

The use of pump therapy in smaller children has also been reported. However, in these cases, closer supervision is required, mainly in relation to the manipulation of the pump. ${ }^{18-21}$

Before indicating pump therapy, the subject should be discussed openly and in detail with the family and the patient. The aspects relative to the operation of the pump, the results that are expected of this therapeutic modality, the complications that may arise and the costs of the treatment should be approached in a realistic way. The dropout rate varies with age and sex, but tends to be greater with patients that are less well-informed regarding the treatment. 22

If the family and the patient decide to use pump infusion, training should be provided in carbohydrate counting, which is essential to determine the insulin dose to be infused before food ingestion. A period of experience with infusion of saline solution should also be offered to the patient, for training on how to operate buttons and change catheter, reservoir and other infusion 22 supplies. After the patient and the family are properly trained, the use of insulin pump therapy begins.

Since insulin pump therapy improves insulin absorption, the daily total dose of insulin tends to decrease. The total dose used by the patient should be reduced in 20 to $30 \%$ with the use of the pump. 
The daily total dose of insulin is then divided between the basal infusion and the pre-meal boli, usually in the proportion of $50 \%$ for basal infusion and $50 \%$ for boli. ${ }^{23}$

The basal infusion dose is given along 24 hours, initially using just one infusion speed, which will later be adjusted as required. The remaining bolus amount will be divided between the meals, based on the eating habits and the amount of carbohydrates ingested in each meal. 23

\section{Indications for pump therapy}

The increased use of insulin pump therapy and most importantly the positive results obtained may lead to the impression that this treatment modality should be universal. Although virtually all patients with type 1 diabetes can use insulin pump therapy, not all will necessarily improve metabolic control with this technology.

Several reports on pump use show that the initial reduction in glycated hemoglobin levels is reversed with time, so that after 6 months to 1 year, the levels are similar to those recorded immediately before the pump. 24-26

An analysis of the case series of the Canadian center with the largest experience in pump infusion therapy shows the need to elaborate a specific program to followup patients using the pump. Treatment adhesion and, above all, the care in calculating the doses, carbohydrate counting and observance of boli, should be monitored by a program providing multiprofessional support, aimed specifically at diabetic patients using infusion pump therapy. 27

There are no lists of indications in the literature for the use of pump infusion therapy. Not even the American Association of Diabetes, which develops most of the clinical guidelines, has a list of indications, making it clear that the decision to use the pump should be made on an individual basis, as is the case with several other aspects of diabetes treatment. ${ }^{28}$

\section{The patient using the pump}

Many patients and their family believe that the pump will minimize glycemic control. This is not the case and therefore should not be a reason to use the pump. Capillary glucose should be measured in the morning in fasting, before and after meals and at least once a week between 2 and 4 a.m.

Since basal insulin levels are maintained in a plateau, without action peaks as occurs with intermediate acting insulins (NPH or L), there is no need to eat snacks. However, every time the patient eats, s/he should measure capillary glucose and infuse an additional insulin bolus. The adjustment of basal insulin is based on capillary glucose measurements in fasting, at dawn and before the meals. The bolus dose is adjusted based on post-meal capillary glucose.23,29

The aim of glycemic control should be individualized for each patient, but pre-meal and fasting glucose levels should be kept between 70 and $120 \mathrm{mg} / \mathrm{dL}$, whereas postmeal levels should not exceed $180 \mathrm{mg} / \mathrm{dL}$.

The infusion device (reservoir, catheter and needle) should be changed every 3 to 5 days, or whenever the metabolic control worsens, with high levels of capillary glucose. 23,29 The child or adolescent should maintain their daily activities, especially the practice of sports. In the case of water sports, although the device can become wet, it cannot be submerged. In that case, the pump should be disconnected with the needle remaining inserted, without infusion of insulin during that period. Contact sports can be practiced with the pump being connected or not, depending on the situation. During the practice of sports, the period without insulin infusion and the ingestion or not of food depends on the type and length of the activity, as well as on age and sex.

Although the current devices have several alarms, malfunction of the pump can occur. In that case, since the action time of the insulin that is being infused is limited, metabolic imbalance, including metabolic ketoacidosis, will occur usually within a few hours.

Metabolic imbalance can be controlled if the malfunction is resolved with the application of a corrective insulin bolus using the same pump. If the pump cannot be fixed, fast or ultrafast insulin should be delivered with a syringe or insulin pen. ${ }^{29}$ In the presence of metabolic ketoacidosis, the use of the pump should be suspended, and the ketoacidosis should be treated according to the routine of the clinic. After the situation is under control, the pump should be reconnected, the cause (infection, malfunction of pump, insulin omission or overeating) having been clarified and solved. 29

When the pump malfunction is detected before the onset of metabolic imbalance, intermediate or slow-acting insulin (glargine or detemir) should be administered. This will have the same effect as the basal insulin and pre-meal bolus application administered with the functioning pump.

With intermediate-acting insulin (NPH), the total dose administered in the last 24 hours should have an additional $20 \%$ given in two daily applications until the new device is obtained. If slow-acting insulin is used, glargine or detemir should be used for the total basal dose with ultrafast insulin boli.

\section{Pump infusion vs. multiple insulin doses}

Some variables should be discussed independently to address the issue of whether treatment with multiple doses is better or worse than pump infusion therapy. 
Initially, one of the most important aspects to be evaluated is the possibility of improving metabolic control.

Several studies sought to compare these two modalities of diabetes treatment. Most of these studies were performed with adults in the 1990s, when technical difficulties prevailed in the use of these pumps and of continuous glucose monitors.

Some studies showed an improvement in metabolic control determined by both mean capillary glucose levels and glycated hemoglobin levels. These studies focused on the adult population, and only a few used randomization techniques.

Two meta-analyses were published within one year. Although each covers a different number of articles from the same data base, both conclude that pump therapy improved metabolic control. The first meta-analysis of 13 reports, published in March 2002 by Pickup et al., showed that pump infusion therapy promoted a small improvement in of the levels of both capillary glucose and glycated hemoglobin. 30,31 The second meta-analysis of 52 reports, published in April 2003 by Weissber-Benchell et al., showed a more important improvement in the same parameters in the group receiving pump infusion ${ }^{32}$ therapy.

In other parameters, the two meta-analyses were in agreement with other reports in the literature: pump infusion therapy reduced the insulin dose, was associated with less variability of capillary glucose results, and users reported improvement in quality of life scores.

Another factor taken into account was the possibility of weight gain, which can happen with both modalities of intensive treatment.

A study with 23 type 1 diabetes patients with ages ranging from 9 to 14 years randomly compared pump infusion therapy with insulin lispro to treatment with multiple doses using NPH insulin and regular insulin. After 3.5 months, the groups switched treatments, with the one using a pump moving to multiple doses and vice- versa. ${ }^{33}$ No differences were found in terms of metabolic control, insulin dosage, weight gain, number of hypoglycemic events, number of metabolic ketoacidosis episodes or infection in insulin application sites.

Patients on pump infusion therapy had better quality of life scores and were more satisfied with the treatment as measured by specific questionnaires. At the end of the study, 16 patients chose to maintain pump infusion therapy.

A Brazilian study carried out in the state of Ceará with adolescents and young adults showed improvement of glucose control in type 1 diabetes patients after the change from conventional treatment or multiple doses to pump infusion therapy. ${ }^{34}$

The need for intensive treatment in diabetes mellitus is clear. Currently, this is done so as to avoid the fearsome complications of this chronic disease. However, it should be emphasized that even if pump infusion therapy is considered the gold-standard of insulin therapy, ${ }^{35}$ the application of multiple insulin doses with a basal/bolus scheme has shown results that are almost as good as those obtained with the pump, ${ }^{36}$ especially in more stable patients. As such, the pump should not be indicated to all diabetic patients, but rather to selected patients for whom medium and long term complications may be reduced.

\section{Cost of treatment}

Regarding the cost of treatment, the price of the device and of the necessary supplies should be taken into account. The system required to start the treatment, including the pump, catheters, syringes, needles, infusers, material for inserting the catheter and fixation belts costs about US\$5,000.37

In addition to this initial cost, the maintenance of treatment involves annual spending of about US $\$ 1,500$ with pump supplies, insulin, capillary glucose strips, batteries and other requirements. 37

In several countries, especially in North America, that cost can be amortized and is at least partially covered by health plans. In addition, a trial period with the pump can be negotiated with some manufacturers before the definitive purchase.

When compared to the other forms of insulin administration, pump infusion therapy is more expensive. The annual cost of pump infusion therapy in the USA, not counting amortization of the device, ranges from US\$ 2,274 to US\$ 4,234 depending on the brand.

The cost of insulin pens also varies according to brand: between US\$2,938 and US\$ 4,296. The cost of using syringes is lower, between US\$ 1,008 and US $\$ 1,495$ per year. 37

A study carried out in England aimed to evaluate the cost-effectiveness of pump infusion therapy. ${ }^{38}$ Taking into account the cost of the pump and supplies, pump therapy would translate into a US\$1,250 increase in the annual cost of the treatment, even considering the savings resulting from improvement of metabolic control reduced number of hypoglycemic episodes and reduced use of insulin. The study did not establish the economic impact associated with improvement in quality of life, because the only data concerning this aspect had been supplied by one of the manufactures of insulin pumps.

That study ${ }^{38}$ considers that the cost of pump therapy represents an effective advantage in case of failure of multiple-dose therapy, to achieve good metabolic control.

It is important to emphasize that this therapeutic modality should only be introduced by a trained team. The patient should be correctly advised and remain under 
close follow-up by a specialized team, with easy access to team members, so that any problems appearing can be solved promptly and efficiently. 38,39

\section{Complications associated with the pump}

The report of complications associated with the infusion system and pump operation was more frequent in the 1980s, having disappeared from the literature in the past years. Problems such as obstruction of the catheter, development of infections at the infusion site, malfunction or acceleration of the infusion rate were gradually solved with the introduction of new materials, new types of insulin, rotation of infusion site and improvement of electronic components. 40

More recent reports emphasize the concern with the fast onset of metabolic ketoacidosis, which may result from the failure to pay attention to equipment alarms signaling absent or interrupted infusion, leading to metabolic imbalance. Since the action of the insulin infused is of short duration, not much time is required for the onset of metabolic imbalance. 41

There have been reports of trophic alterations (lipoatrophy) associated mainly with the insulin used, and not so much with the pump itself. ${ }^{42}$ This problem was significantly minimized with the use of highly purified forms.

It has been suggested that pump therapy leads to weight gain. However, more recent studies show that weight control becomes actually easier, since the use of basal/bolus doses of insulin promotes a balance between the amount of food ingested, insulin dose and physical activity. 43

\section{Future developments}

A continuous glucose monitoring system that is currently under development measures glucose during several consecutive days (CGMS ${ }^{\circledR}$ Medtronic MiniMed, Northridge, CA) using a glucose oxidase-plated platinum electrode inserted into subcutaneous tissue. The system stores consecutive glucose measurements for later analysis. 44

A similar device (Guardian, Medtonic MiniMed) which has been tested provides real-time glucose measurements which are be transmitted around the clock to a glucose sensor using radio frequency (wireless).

It seems that the ideal "closed-loop" system, in which there is real-time communication with an infusion pump that relies on either a portal or peripheral venous insulin delivery system, providing just the right amount of insulin for the maintenance of normal glucose levels, is not that far from becoming a reality. 45

\section{Conclusions}

Insulin pump therapy is a safe and effective alternative for type 1 diabetes patients, resulting in improved metabolic control. Nevertheless, the greatest advantage of this therapeutic modality is the reduction in hypoglycemic events and the greater freedom in terms of lifestyle and eating habits, although the need capillary glucose measurements remain.

The cost of insulin pump therapy is the highest among the therapeutic modalities available. In the specific situations in which this treatment modality is cost-effective, it should be the therapy of choice.

\section{References}

1. The effect of intensive treatment of diabetes on the development and progression of long-term complications in insulin-dependent diabetes mellitus. The Diabetes Control and Complications Trial Research Group. N Engl J Med. 1993;329:977-86.

2. Intensive blood glucose control with sulphonylureas or insulin compared with conventional treatment and risk of complications in patients with type 2 diabetes (UKPDS 33). UK Prospective Diabetes Study (UKPDS) Group. Lancet. 1998;352:837-53.

3. Lenhard MJ, Reeves GD. Continuous subcutaneous insulin infusion. Arch Intern Med. 2001;161:2293-300.

4. Maniatis AK, Klingensmith GJ, Slover RH, Mowry CJ, Chase HP. Continuous subcutaneous insulin infusion therapy for children and adolescents: an option for routine diabetes care. Pediatrics. 2001;107:351-6.

5. Bode BW, Sabbah HT, Gross TM, Fredrickson LP, Davidson PC. Diabetes management in the new millennium using insulin pump therapy. Diabetes Metab Res Rev. 2002;18:S14-20.

6. Tamborlane WV, Sherwin RS, Genel M, Felig P. Reduction to normal of plasma glucose in juvenile diabetes by subcutaneous administration of insulin with a portable infusion pump. $\mathrm{N}$ Engl J Med. 1979;300:573-8.

7. Marcus AO, Fernandez MP. Insulin pump therapy. Postgrad Med. 1996;99:125-32.

8. Plodkowski RA, Edelman SV. The state of insulin pump therapy:2002. Curr Opin Endocrinol Diabetes. 2002;9:329-37.

9. Raskin $\mathrm{P}$, Holcombe JH, Tamborlane WV, Malone JI, Strowig S, Ahern JA, et al. A comparison of insulin lispro and buffered regular human insulin administered via continuous subcutaneous insulin infusion pump. J Diabetes Complications. 2001;15: 295-300.

10. Renner R, Pfutzner A, Trautmann M, Harzer O, Sauter K, Landgraf $R$. Use of insulin lispro in continuous subcutaneous insulin infusion treatment. Diabetes Care. 1999;22:784-8.

11. Wolpert HA, Faradji RN, Bonner-Weir S, Lipes MA. Metabolic decompensation in pump users due to lispro insulin precipitation. BMJ. 2002;324:1253.

12. Bode B, Weinstein R, Bell D, McGill J, Nadeau D, Raskin P, et al. Comparison of insulin aspart with buffered regular insulin and insulin lispro is continuous subcutaneous insulin infusion. Diabetes Care. 2002;25:439-44.

13. Reichel A, Rietzsch H, Kohler HJ, Pfutzner A, Gudat U, Schulze J. Cessation of insulin infusion at night-time during CSIItherapy; comparison of regular human insulin and insulin lispro. Exp Clin Endocrinol Diabetes. 1998;106:168-72.

14. American Diabetes Association. Continuous subcutaneous insulin infusion. Diabetes Care. 2003;26:S125.

15. Steindel BS, Roe TR, Costin G, Carlson M, Kaufman FR. Continuous subcutaneous insulin infusion in children and adolescents with chronic poorly controlled type 1 diabetes mellitus. Diabetes Res Clin Pract. 1995;27:199-204.

16. Tamborlane WV, Bonfig W, Boland E. Recent advances in the treatment of youth with type 1 diabetes: better care through technology. Diabet Med. 2001;18;864-70. 
17. DeVries JH, Snoek FJ, Kostense PJ, Masurel N, Heine RJ, Dutch Insulin Pump Study Group. A randomized trial of continuous subcutaneous insulin infusion and intensive injection therapy in type 1 diabetes for patients with long-standing poor glycemic control. Diabetes Care. 2002;25:2074-80.

18. Ahern JA, Boland EA, Doane $R$, Ahern JJ, Rose $P$, Vincent $M$, et al. Insulin pump therapy in pediatrics: a therapeutic alternative to safely lower HbA1c levels across all age groups. Pediatr Diabetes. 2002:3:10-5.

19. Plotnick LP, Clark LM, Brancati FL, Erlinger T. Safety and effectiveness of insulin pump therapy in children and adolescents with type 1 diabetes. Diabetes Care. 2003;26:1142-6.

20. Klingensmith GJ, Temple-Trujillo R, Johnson D. Pump therapy for children: weighing the risks and benefits. Diabetes Spectr. 2001; 14:81-3.

21. Litton J, Rice A, Friedman N, Oden J, Lee MM, Freemark M. Insulin pump therapy in toddlers and preschool children with type 1 diabetes mellitus. J Pediatr. 2002;141:490-5.

22. Conrad SC, McGrath MT, Gitelman SE. Transition from multiple daily injections to continuous subcutaneous insulin infusion in type 1 diabetes mellitus. J Pediatr. 2002;140:235-40.

23. Farkas-Hirsch R, Hirsch IB. Continuous subcutaneous insulin infusion: a review of the past and its implementation for the future. Diabetes Spectr. 1994;7:80-4.

24. Artiles-Sisk A, Buccino J, Pearlman K, Danemann D and the Diabetes Team. Pump therapy in children and teens with type 1 diabetes. Can J Diabetes Care. 2001;25:89A.

25. Weintrob N, Benzaquen $H$, Shalitin S, Fayman G, Galatzer A, Dickerman $Z$, et al. Continuous subcutaneous insulin infusion versus multiple daily injections in children with type 1 diabetes. Diabetologia. 2001;44:A26.

26. Howard CP, Pettis D, Schurig JM. Use of continuous subcutaneous insulin infusion therapy in a pediatric type 1 diabetes mellitus population. Diabetes. 2000;49:A359.

27. Liberatore R Jr., Pearlman K, Buccino J, Artiles-Sisk A, Daneman D. Continuous subcutaneous insulin infusion pump treatment in children with type 1 diabetes mellitus. J Pediatr Endocrinol Metab. 2004;17:223-6.

28. Silverstein J, Klingensmith G, Copeland K, Plotnick L. Kaufman $F$, Laffel $L$, et al. Care of children and adolescents with type 1 diabetes: a statement of the American Diabetes Association. Diabetes Care. 2005;28:186-212.

29. Boland EA, Grey M, Oesterle A, Fredrickson L, Tamborlane WV. Continuous subcutaneous insulin infusion. A new way to lower risk of severe hypoglycemia, improve metabolic control and enhance coping in adolescents with type 1 diabetes. Diabetes Care. $1999 ; 22: 1779-84$

30. Pickup J, Keen $\mathrm{H}$. Continuous subcutaneous insulin infusion at 25 years. Evidence base for the expanding use of insulin pump therapy in type 1 diabetes. Diabetes Care. 2002;25:593-8.

31. Pickup J, Mattock M, Kerry S. Glycaemic control with continuous subcutaneous insulin infusion compared with intensive insulin injections in patients with type 1 diabetes; meta-analysis of randomised controlled trials. BMJ. 2002;324:705.

32. Weissberg-Benchell J, Antisdel-Lomaglio J, Seshadri R. Insulin pump therapy. A meta-analysis. Diabetes Care. 2003;26: 1079-87.
33. Weintrob $\mathrm{N}$, Benzaquen $\mathrm{H}$, Galatzer A, Shalitin $\mathrm{S}$, Lazar $\mathrm{L}$ Fayman G, et al. Comparison of continuous subcutaneous insulin infusion and multiple daily injection regimens in children with type 1 diabetes: a randomized open crossover trial. Pediatrics. 2003;112:559-64.

34. Hissa $\mathrm{MH}$, Hissa AS, Bruin VM, Fredrickson LP. Comparison between continuous subcutaneous insulin infusion and multiple insulin injection therapy in type 1 diabetes mellitus; 18 -month follow-up. Endocr Pract. 2002;8:411-6.

35. Renard E. Intensive insulin therapy today: "basal-bolus" using multiple daily injections or CSII? Diabetes Metab. 2005;31:4S404 S44.

36. Alemzadeh R, Palma-Sisto P, Parton EA, Holzum MK. Continuous subcutaneous insulin infusion and multiple dose of insulin regimen display similar patterns of blood glucose excursions in pediatric type 1 diabetes. Diabetes Technol Ther. 2005;7:587-96.

37. Kanakis SJ, Watts C, Leichter SB. The business of insulin pumps in diabetes care; clinical and economic considerations. Clinical Diabetes. 2002;20:214-6.

38. National Institute for Clinical Excellence. Guidance on the use of continuous subcutaneous insulin infusion for diabetes. Technol Appraisal Guidance. 2003;57:1-23.

39. Feltbower RG, Campbell FM, Bodansky HJ, Stephenson CR, McKinney PA. Insulin pump therapy in childhood diabetes-cost implications for Primary Care Trusts. Diabet Med. 2006;23:86-9.

40. Mecklenburg RS, Benson EA, Benson JW Jr., Fredlund PN, Guinn $T$, Metz RJ, et al. Acute complications associated with insulin infusion pump therapy. JAMA. 1984;252:3265-9.

41. Mack-Fogg JE, Orlowski CC, Jospe N. Continuous subcutaneous insulin infusion in toddlers and children with type 1 diabetes mellitus is safe and effective. Pediatr Diabetes. 2005;6:17-21.

42. Griffin ME, Feder A, Tamborlane WV. Lipoatrophy associated with lispro insulin in insulin pump therapy. Diabetes Care. $2001 ; 24: 174$

43. Raile K, Noelle V, Landgraf R, Scharz HP. Weight in adolescents with type 1 diabetes mellitus during continuous subcutaneous insulin infusion therapy. J Pediatr Endocrinol Metab. 2002;15: 607-12.

44. Gross TM, Bode BW, Einhorn D, Kayne DM, Reed JH, White NH, et al. Performance evaluation of the MiniMed ${ }^{\circledR}$ continuous glucose monitoring system during patient home use. Diabetes Technol Ther. 2000;2:49-56.

45. Steil GM, Panteleon AE, Rebrin K. Closed-loop insulin delivery the path to physiological glucose control. Adv Drug Deliv Rev. $2004 ; 56: 125-44$

Correspondence:

Raphael Del Roio Liberatore Jr.

Rua Ondina, 54

CEP 15015-205 - São José do Rio Preto, SP - Brazil

E-mail: liberat@famerp.br 